Results Of 169 children, 139 (82\%) females. Median age at disease onset:11.4 years(3.4-18), median age at diagnosis:12 (3.5-19).

$20 \%$ had history of autoimmune disease in first degree relative.

Therapy at disease onset(first 6 months):

Hydroxychloroquine: 100\%,

Glucocorticoids: 98\%,

Mycophenolate: 33\%,

Methotrexate: 27\%,

Azathioprine: 16\%,

Cyclophosphamide: 9\%,

Rituximab: 2\%.

At last follow up: Glucocortocoids: $37 \%$

Median follow up 48 months(1-195 months), Mortality:4\%,lost to follow up: $19 \%$, active disease at last follow up: $25 \%$

Conclusions Patients seen at our centre had a significant disease burden with a median SLEDAI score of $>20$ at presentation.

Upto $1 / 2$ of the study population did not have a malar rash. 38\% had renal disease. Fever was seen in $82 \%$ and often was the cause for seeking medical opinion. This is a small data set from a tertiary level centre and not representative of the community disease.

\section{RITUXIMAB IN SLE MEMBRABOUS NEPHROPATHY}

${ }^{1} \mathrm{R}$ Sinha*, ${ }^{2} \mathrm{~S}$ Akhtar, ${ }^{2} \mathrm{P}$ Giri, ${ }^{2} \mathrm{P}$ Pal. ${ }^{1}$ Kolkata, India; ${ }^{2}$ Institute of Child Health, Paediatric Nephrology, Kolkata, India

\subsection{6/lupus-2017-000215.143}

Background and Aims We hereby report three cases of biopsy proven Type 5 SLE membranous nephropathy (T5: SLEMN) which responded to Rituximab after failed first line induction agents.

Methods Retrospective chart review

Results Case 1: Rituximab was used in this 14 year old girl as she failed to standard therapy of cyclophosphamide $(750 \mathrm{mg} /$ $\mathrm{m}^{2}$ ) and high dose corticosteroid along with angiotensin convertase inhibitor (ACEI). 30 days post rituximab 24 hour proteinuria dropped (6790 to $876 \mathrm{mg}$ ) and albumin rose $(1.8 \mathrm{mg} /$ $\mathrm{dl}$ to $3 \mathrm{mg} / \mathrm{dl}$ ). She is in remission at 14 month on low dose steroid and mycophenolate mofetil (MMF).

Case 2: 10 year old girl whose presentation was similar to Case 1 and failed to show any significant improvement tostandard therapy. 45 days post rituximab, 24 hour proteinuria dropped (4900 to $690 \mathrm{mg}$ ) and albumin rose $(1.9 \mathrm{mg} / \mathrm{dl}$ to $3.3 \mathrm{mg} / \mathrm{dl}$ ). At 12 month she is in remission on low dose steroid and MMF.

Case 3: 12 year old girl presented with features of nephrotic syndrome. At 30 days follow up there were no improvement despite standard therapy and she also started to have neuropsychiatric manifestation. 60 days post rituximab 24 hour proteinuria dropped (3548 to $300 \mathrm{mg}$ ) and albumin rose $(1.8 \mathrm{mg} / \mathrm{dl}$ to $3.7 \mathrm{mg} / \mathrm{d}) 1$. Her neuro-psychiatric manifestation also improved.

Conclusions In all 3 cases 2 doses of rituximab at $375 \mathrm{mg} / \mathrm{m}^{2}$ each achieved CD 19 count of zero (which normalised by 14 , 9 and 11 month respectively) along with significant drop in proteinuria.

\section{4 ROLE OF TUBULOINTERSTITIAL LESIONS IN PREDICTING RENAL OUTCOME AMONG PAEDIATRIC ONSET LUPUS NEPHRITIS - A RETROSPECTIVE COHORT STUDY}

${ }^{1} \mathrm{CY} \mathrm{Wu}{ }^{*},{ }^{2} \mathrm{HP}$ Chien, ${ }^{3} \mathrm{HY}$ Yang, ${ }^{4} \mathrm{MH}$ Tseng, ${ }^{1} \mathrm{KW}$ Yeh, ${ }^{1} \mathrm{JL}$ Huang. ${ }^{1}$ Chang-Gung Memorial Hospital- Linko branch, Division of Allergy- Asthma- and Rheumatology- Department of Paediatrics, Taoyuan city, Taiwan R.O.C; ${ }^{2}$ Chang-Gung Memorial Hospital- Linko branch, Department of Pathology, Taoyuan city, Taiwan R.O.C; ${ }^{3}$ Chang-Gung Memorial HospitalLinko branch, Department of Nephrology, Taoyuan city, Taiwan R.O.C; ${ }^{4}$ Chang-Gung Memorial Hospital- Linko branch, Division of Paediatric Nephrology- Department of Paediatrics, Taoyuan city, Taiwan R.O.C

\subsection{6/lupus-2017-000215.144}

Background and aims Raising evidence supported a prognostic utility of tubulointerstitial lesions in lupus nephritis (LN). The exact prevalence of tubulointerstitial abnormalities and its predictive value among paediatric onset systemic lupus erythematous (pSLE) cases, however, remained unknown.

Methods Sixty-seven pSLE subjects diagnosed with LN with initial renal samples available were enrolled and followed for an average of $6.43 \pm 3.06$ years. Renal histology was evaluated according to the International Society of Nephrology/Renal Pathology Society classification, National Institute of Health classification and tubulointerstitial activity index (TIAI).

Results Tubulointerstitial injuries were observed in $38.81 \%$ of all LN cases, including $13.33 \%$ with non-proliferative lupus nephritis (nPLN) and $46.15 \%$ of with proliferative lupus nephritis (PLN). Tubulointerstitial injuries occurred solitarily in cases with nPLN(13.33\%), but always associated glomerular changes and significantly impacted renal survival $(\mathrm{p}=0.032)$ among those with PLN. TIAI associated glomerular abnormalities $(p=0.031)$ but did not correlate renal performance or subsequent outcome $(p=0.445)$. Among the chronicity index, it was the chronic tubulointerstitial lesions which provided prognostic information $(\mathrm{p}=0.012)$. We observed a synergistic effect of all tubulointerstitial abnormalities rather than an individual factor attributed the prognostic utility $(p=0.025$ vs. $\mathrm{p}=0.083,0.055,0.354$ ). Finally, considering tubulointerstitial injuries in PLN further discriminated subsequent renal outcome $(p=0.006)$.

Conclusions The prevalence and clinical significance of tubulointerstitial abnormalities were similar among the pSLE and the adult population. With its importance in identifying those at risk of renal failure, histologic classification considering tubulointerstitial lesions may potentially assist outcome prediction.

\section{Patient-submitted abstracts}

\section{A PATIENT'S FOUR DECADE JOURNEY TO WELLNESS : A MODEL OF CARE FOR LIVING WELL WITH LUPUS}

M Erceg*. ${ }^{\text {Perth, Australia }}$

\subsection{6/lupus-2017-000215.145}

Background and aims Mary Erceg is a former teacher and senior public servant who has lived with systemic lupus erythematosus for over 40 years since initial diagnosis.

This presentation explores her personal journey through initial diagnosis; medications; flares; acute relapses; and treatment errors which resulted in 6 days in a coma, four months 